\section{Human visual motion sensitivity: Evidence against a ratio theory of sensory coding}

\author{
ROBERT SEKULER, DONALD LEHR, WILLIAM STONE, and MARK WOLF \\ Northwestern University, Evanston, Illinois 60201
}

Luminance thresholds for downward moving contours were measured under several conditions of adaptation. Included was one condition which desensitized visual mechanisms responsive to downward motion. Another condition exerted equal effects on both up. and down-sensitive mechanisms. Thresholds for moving contours were unaffected by exposure to contours which moved in the opposite direction. This indicates that the perception of motion does not depend upon the relative activity in oppositely tuned, directionally selective visual mechanisms.

Neural cells in the visual systems of various species respond selectively to the direction with which contours move through the visual field (Jacobs, 1969). Some of the properties of human motion perception have been attributed to analogous, directionally selective mechanisms in our own visual systems (Over, in press; Hepler, 1968; Weisstein, 1969). A major component of attempts to establish such parallels is an explicit hypothesis about the principles of sensory coding used by these putative human motion-analyzers. One explicit theory of such sensory coding was offered by Sutherland (1961) and has been adopted, without formal test, as a working assumption by others (Sekuler \& Ganz, 1963; Sekuler \& Pantle, 1967; Cormack, 1964; Barlow \& Hill, 1963). The present paper shows that while Sutherland's theory may hold for special phenomena of motion perception, it is inadequate for other, more general phenomena.

Sutherland's theory may be characterized as a "ratio theory" since it states that the perception of motion depends upon the ratio of activities in two sets of sensory mechanisms or "analyzers." For the case of motion downward through the field, we may represent Sutherland's theory in the following way:

$$
\begin{aligned}
& \text { Percept "down" } \\
& =\mathrm{f}\left[\frac{\text { (response of down-analyzers) }}{\text { (response of up-analyzers) }}\right]
\end{aligned}
$$

(Expression 1)

This expression implies that the $S$ experiences downward motion whenever visual mechanisms normally sensitive to motion down are more active than mechanisms normally sensitive to motion up. This ratio theory was originally designed to explain the waterfall illusion or motion aftereffect ${ }^{1}$ and is consistent with the known properties of that aftereffect.

In order to test the ratio theory's generality, we must examine its implications for phenomena other than the motion aftereffect. One such implication is that adaptation of down-analyzers, via prolonged inspection of downward-moving contours, will render motion in the downward direction less visible. This may be conceptualized as a reduction in the potential size of the numerator of Expression 1 (above). Exposure to adaptation contours moving in one direction does in fact raise the luminance threshold for contours which move in the same direction. But this result by itself cannot validate the ratio theory, since it could have been due to a loss of sensitivity restricted to one class of directional analyzer without the mediation of the ratio per se.

\section{METHOD}

We have tried to test the ratio theory directly. If, as that theory states, the perception of downward motion depends upon the relative response of down- and up-analyzers, we should expect any treatment which reduces the sensitivity of both sets of analyzers by the same amount to have no effect on the threshold for downward-moving contours. This treatment, one among several we studied, can be thought of as reducing both the numerator and the denominator of the sensory coding ratio by the same amount-leaving the ratio unaffected.

All stimulation was delivered in monocular Maxwellian view, using an optical system fully described elsewhere (Pantle \& Sekuler, 1969). Method of adjustment was used to measure the luminance threshold for a low spatial frequency square-wave grating $(0.38 \mathrm{c} / \mathrm{deg})$ of horizontal bars which moved downward at $5 \mathrm{deg} / \mathrm{sec}$. This test target was presented within a 6-deg-diam field concentric with and optically superposed on a larger, 12-deg-diam uniform background of $36 \mathrm{~cd} / \mathrm{m}^{2}$. Thresholds for this downward-moving grating were measured after exposure to each of four different types of adaptation fields. In three of the adaptation conditions the $S$ saw a high-contrast (1.00) grating of $0.38 \mathrm{c} / \mathrm{deg}$ presented within a 12-deg-diam field. The space-average luminance of the adaptation field was equal to that of the background for the test stimulus, $36 \mathrm{~cd} / \mathrm{m}^{2}$. After the $\mathrm{S}$ had been allowed to view the adaptation field for $4 \mathrm{~min}$, a series of alternating test and adaptation periods began. During or immediately after each brief test period, the $S$ adjusted the luminance of the test target. When the $\mathrm{S}$ was finally satisfied that threshold luminance had been established, it was recorded and the trial terminated. That final luminance value defined the threshold for the trial. A small dark central fixation point was present throughout.

Thresholds for downward-moving test contours were measured under four conditions of adaptation. Each condition consisted of two components which alternated repeatedly. These components differed from condition to condition in such a way that comparisons among thresholds for the downward-moving test contours measured in each of the conditions would reveal the differential contributions of various components. In one condition, designed to affect only down-sensitive mechanisms, two equally long periods of downward adaptation motion alternated, separated by a short dark interval. This condition will be referred to as down-down or D-D. The second condition, hereafter referred to as stationary-down or S-D, consisted of alternating periods of stationary contours and downward-moving contours. Since this condition contained downward-moving contours for only a fraction of its total cycle time, we would expect some dissipation of the adaptation during the stationary portion of the cycle produced by the downward-moving contours. As a result, differences between the threshoids for downward-moving test contours in Conditions D-D and S-D reflect the amount of direction-specific adaptation that dissipated during the stationary portion of the S-D cycle. A third adaptation condition, up-down, or U-D, was designed to affect both up-sensitive and down-sensitive mechanisms equally. It resembled Condition S-D except that it involved alternating periods of upward-moving and downward-moving adaptation contours. Conditions U-D and S-D had the same fraction of their cycles given to downward adapting motion. Differences in the thresholds for downward-moving test contours measured in those two conditions then would reflect 


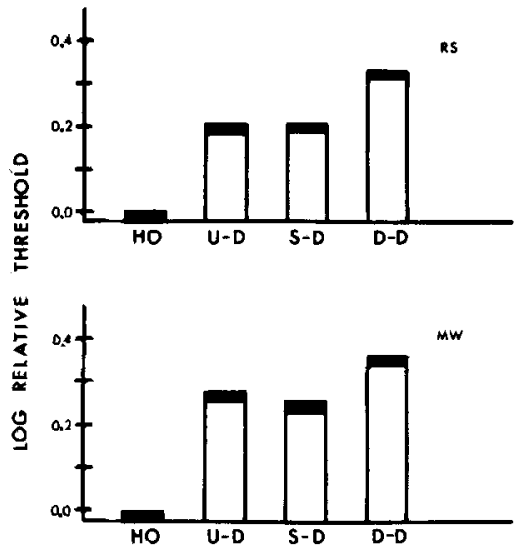

Fig. 1. Log relative thresholds for a downward-moving grating in each of four conditions: HO (a uniform adaptation field); U-D, alternating down- and up-moving adaptation grating; and D-D, down-moving adaptation grating alternated with another down-moving grating. Upper panel, S RS; lower panel, S MW. Thresholds are scaled relative to $\mathrm{HO}$ as a zero level. Darkened areas in each bar indicate one standard error of the mean down from the mean.

the differential contributions of upward-moving vs stationary adaptation contours. A final condition provided a control for the effects of light adaptation upon the threshold for the test contours. In this condition, a uniform field of equivalent space-average luminance was presented in place of the adaptation contours of the other conditions. In this condition, too, the periodic brief dark intervals were retained in the cycle to insure comparability.

In all conditions involving motion of the adaptation contours the velocity was the same, $8 \mathrm{deg} / \mathrm{sec}$. Each portion of an adaptation cycle, except the dark intervals, was $2.0 \mathrm{sec}$ in duration. The periodic dark intervals lasted $0.5 \mathrm{sec}$. As indicated above, adaptation periods were presented in a continuously recycling manner. In all four adaptation conditions, a test period occurred once every $12 \mathrm{sec}$. During this period the test target and background were presented for $0.75 \mathrm{sec}$. The test period allowed the $S$ to adjust the luminance of the downward-moving test target, bringing it closer to threshold. All trials began with the test target at a randomly chosen suprathreshold luminance level. Because the total duration of a given adaptation cycle $(5 \mathrm{sec})$ did not bear an integral relationship to the duration $(12 \mathrm{sec})$ of the test cycle, test periods occurred randomly with respect to the various components of any adaptation cycle. For example, in the U.D condition, the test period occurred just about as often after an upward-moving part of the cycle as after a downward part of the cycle.

\section{RESULTS AND DISCUSSION}

Each of two Ss was tested, in a random order, six times with each of the four conditions of adaptation. The testing for each $S$ was distributed over several days to minimize long-term interactions among conditions. The results are shown in Fig. 1 , where the mean log relative threshold for each $S$ and condition are given. The absolute values of the thresholds for S MW tended to be approximately $0.1 \mathrm{log}$ units below those for RS but, in Fig. 1, data for each $S$ has been scaled relative to his own threshold for the homogeneous control condition. The shaded area on each bar of the bar graph indicates one standard error of the mean for that condition, down from the mean.

The pattern of results for both $\mathrm{Ss}$ is much the same, and any differences between conditions that will be discussed here are statistically significant at the .01 level or better. ${ }^{2}$ Relative to the homogeneous adaptation condition, thresholds are elevated in the other three conditions of adaptation. This shows that viewing downward-moving contours raises the threshold for contours which move in that same direction. The D-D condition, however, produces a larger threshold elevation than either the U-D or the S-D condition. Moreover, the latter two conditions, alternating up-down and alternating up-stationary, produce nearly identical effects on the threshold for downward-moving bars. This is the critical comparison as far as the ratio theory is concerned. Conditions $\mathrm{L}-\mathrm{D}$ and $S-D$ are matched in that both have periods of down adaptation and give the same opportunity for the down adaptation to dissipate-during the up period, in the case of U-D, and during the stationary period, in the case of S.D. Since Conditions U.D and S-D have identical effects on the threshold for downward motion, we conclude that threshold depends only on the sensitivity of down-analyzers and is unaffected by concomitant desensitization of up-analyzers, as in Condition U-D. The difference between the thresholds in the D-D condition and the $S-D$ condition is an estimate of the extent to which each of the two downward-moving half cycles is contributing to adaptation in the D.D condition.

This experiment demonstrates the difficulty of extrapolating from threshold phenomena, such as direction-specific adaptation, to superthreshold phenomena, such as the motion aftereffect. Many years ago, Wohlgemuth (1911) found that cyclical alternation between opposite directions of adaptation motion yielded almost no net motion aftereffect. Under similar conditions in the present experiment, we found independence of the effects of exposure to opposite directions of adaptation motion. While the motion aftereffect seems to depend upon the relative responsitivity of visual mechanisms sensitive to opposite directions of motion, the threshold for motion in a particular direction depends only upon the sensitivity of mechanisms "tuned" to that direction. This discrepancy should give pause to theorists interested in using psychophysical data to make inferences about the nature of sensory coding (Uttal, in press). Psychophysical phenomena that seem at first to be well correlated may, in fact, reflect quite different operations. 3

\section{REFERENCES}

BARLOW, H. B., \& HILL, R. M. Evidence for a physiological explanation of the waterfall phenomenon and figural aftereffects. Nature, 1963, 200, 1345-1347.

CORMACK, R. H. Visual movement perception and the visual aftereffect of movement. Doctoral dissertation, University of Cincinnati, 1962. University Microfilm No. 62-4775.

HEPLER, N. Color: A motion-contingent aftereffect. Science, 1968, 162, 376-377.

JACOBS, G. H. Receptive fields in visual systems. Brain Research, 1969, 14, 553-573.

OVER, R. Comparison of normalization theory and neural enhancement explanations of negative aftereffects. Psychological Bulletin, in press.

PANTLE, A., \& SEKULER, R. Contrast response of human visual mechanisms sensitive to orientation and direction of motion. Vision Research, 1969, 9, 397-406.

SEKULER, R., \& GANZ, L. Aftereffect of seen motion with a stabilized retinal image. Science, $1963,139,419-420$.

SEKULER, $R_{*}$, \& PANTLE, A. A model for aftereffects of seen movement. Vision Research, 1967, 7, 427-439.

SUTHERLAND, N. S. Figural aftereffects and apparent size. Quarterly Journal of Experimental Psychology, 1961, 13, 222-228.

UTTAL, W. The psychobiological silly season. American Journal of Psy chology, in press.

WEISSTEIN, N. What the frog's eye tells the human brain: Single cell analyzers in the human visual system. Psychological Bulletin, 1969, 72, 157-176.

WOHLGEMUTH, A. On the aftereffect of seen movement. British Journal of Psychology, 1911, Monograph Supplement No. 1.

\section{NoTES}

1. In the motion aftereffect, observation of contours moving in one direction causes a subsequently seen stationary object to appear to move in the opposite direction. According to the ratio theory, viewing contours that move upward reduces the responsivity of up-analyzers. As a result, when stationary contours are presented, the response of previously nonadapted down-sensitive mechanisms will predominate over that of adapted, up-sensitive ones, yielding a percept of upward motion. This interpretation depends upon the assumption that motion analyzers are at least somewhat responsive to stationary contours, an assumption supported by Pantle \& Sekuler (1969).

2. Statistical significance was determined by 1 tests.

3. Supported by EY-00321.

(Accepted for publication November 1, 1970.) 